\title{
Stabilities for a class of higher order integro- differential equations
}

Cite as: AIP Conference Proceedings 2046, 020012 (2018); https://doi.org/10.1063/1.5081532

Published Online: 04 December 2018

L. P. Castro, and A. M. Simões

\section{ARTICLES YOU MAY BE INTERESTED IN}

New convolutions for an oscillatory integral operator on the half-line

AIP Conference Proceedings 2046, 020015 (2018); https://doi.org/10.1063/1.5081535

Convolutions and applications for the offset linear canonical transform via Hermite weights AIP Conference Proceedings 2046, 020014 (2018); https://doi.org/10.1063/1.5081534

On integral operators and equations generated by cosine and sine Fourier transforms AIP Conference Proceedings 2046, 020013 (2018); https://doi.org/10.1063/1.5081533
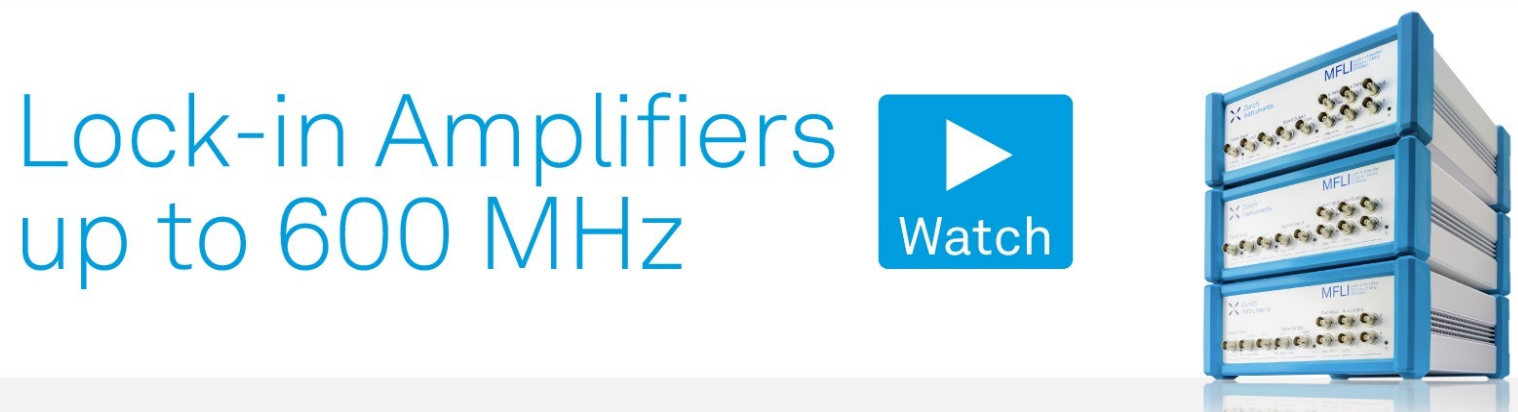


\title{
Stabilities for a Class of Higher Order Integro-Differential Equations
}

\author{
L. P. Castro ${ }^{1, a)}$ and A. M. Simões ${ }^{1,2, b)}$ \\ ${ }^{1}$ Center for Research and Development in Mathematics and Applications (CIDMA), Department of Mathematics, \\ University of Aveiro, Aveiro, Portugal. \\ ${ }^{2}$ Center of Mathematics and Applications of University of Beira Interior (CMA-UBI), Department of Mathematics, \\ University of Beira Interior, Covilhã, Portugal.

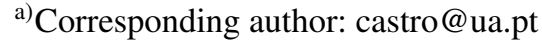 \\ b)asimoes@ubi.pt
}

\begin{abstract}
This work is devoted to analyse different kinds of stabilities for higher order integro-differential equations within appropriate metric spaces. We will consider the $\sigma$-semi-Hyers-Ulam stability which is a new kind of stability somehow between the Hyers-Ulam and the Hyers-Ulam-Rassias stabilities. Sufficient conditions are obtained in view to guarantee Hyers-Ulam, $\sigma$-semiHyers-Ulam and Hyers-Ulam-Rassias stabilities for such a class of integro-differential equations. We will be considering finite and infinite intervals as integration domains. Among the used techniques, we have fixed point arguments and generalizations of the Bielecki metric.
\end{abstract}

\section{INTRODUCTION}

The study of properties associated with stability for functional, differential, integral and integro-differential has been widely diffused and has been a subject of great interest in the last seven decades earning particular interest due to their great number of applications in elasticity, semiconductors, heat conduction, fluid flow, scattering theory, chemical reactions and population dynamic, and others (see $[1,3,6,8,9,10,11,12,16,17,18,20,21,22,23$, 24]). In part, this is because of their potential applicability in mathematical models where we cannot expect to easily obtain the exact solution of the problem but we may expect to obtain an approximate solution which should be stable in a certain specific sense. Hyers-Ulam and Hyers-Ulam-Rassias are among the most studied types of stabilities. These two stabilities were originated from a famous question raised by S. M. Ulam [28] at the University of Wisconsin in 1940, about to know when a solution of an equation differing "slightly" from a given one must be somehow near to the solution of the given equation. A first parcial answer to this question was given by D. H. Hyers, for Banach spaces, in the case of the additive Cauchy equation $f(x+y)=f(x)+f(y)$. It is for this reason that the obtained result is nowadays called the Hyers-Ulam stability. After that preliminary answer, other approaches emerged, and new orientations were introduced by Th. M. Rassias, see [25], introducing therefore the so-called Hyers-Ulam-Rassias stability. Different generalizations were obtained by other researchers, by considering the possibility of using different involved norms and others types of equations, in particular by Aoki [2], Gajda [19], and Rassias [26]. The interested reader can obtain a detailed description of these advances in [4].

In this paper, we study the Hyers-Ulam stability, the $\sigma$-semi-Hyers-Ulam stability and the Hyers-Ulam-Rassias stability for the following class of higher order integro-differential equation,

$$
y^{(n)}(x)=f\left(x, y(x), \int_{a}^{x} k(x, \tau, y(\tau), y(\alpha(\tau))) d \tau\right), \quad y^{(j)}(a)=0, j=0, \ldots, n-1
$$

with $y \in C^{n}([a, b])$, for $x \in[a, b]$ where, for starting, $a$ and $b$ are fixed real numbers, $f:[a, b] \times \mathbb{C} \times \mathbb{C} \rightarrow \mathbb{C}$ and $k:[a, b] \times[a, b] \times \mathbb{C} \times \mathbb{C} \rightarrow \mathbb{C}$ are continuous functions, and $\alpha:[a, b] \rightarrow[a, b]$ is a continuous delay function which fulfills $\alpha(\tau) \leq \tau$ for all $\tau \in[a, b]$. 
The formal definition of the above mentioned stabilities are now introduced for the higher order integro-differential equation (1). If for each continuously differentiable function $y$ satisfying $\left|y^{(n)}(x)-f\left(x, y(x), \int_{a}^{x} k(x, \tau, y(\tau), y(\alpha(\tau))) d \tau\right)\right| \leq \sigma(x), x \in[a, b]$, where $\sigma$ is a non-negative function, there is a solution $y_{0}$ of the higher order integro-differential equation and a constant $C>0$ independent of $y$ and $y_{0}$ such that $\left|y(x)-y_{0}(x)\right| \leq C \sigma(x)$, for all $x \in[a, b]$, then we say that the higher order integro-differential equation (1) has the Hyers-Ulam-Rassias stability.

If for each continuously differentiable function $y$ satisfying $\left|y^{(n)}(x)-f\left(x, y(x), \int_{a}^{x} k(x, \tau, y(\tau), y(\alpha(\tau))) d \tau\right)\right| \leq \theta$, $x \in[a, b]$, where $\theta \geq 0$, there is a solution $y_{0}$ of the higher order integro-differential equation and a constant $C>0$ independent of $y$ and $y_{0}$ such that $\left|y(x)-y_{0}(x)\right| \leq C \theta$, for all $x \in[a, b]$, then we say that the higher order integrodifferential equation has the Hyers-Ulam stability.

We also use a stability in-between the two just mentioned stabilities of Hyers-Ulam-Rassias and Hyers-Ulam, introduced in [14], in the following way:

Definition 1 Let $\sigma$ a non-decreasing function defined on $[a, b]$. If for each continuously differentiable function $y$ satisfying $\left|y^{(n)}(x)-f\left(x, y(x), \int_{a}^{x} k(x, \tau, y(\tau), y(\alpha(\tau))) d \tau\right)\right| \leq \theta, x \in[a, b]$, where $\theta \geq 0$, there is a solution $y_{0}$ of the higher order integro-differential equation (1) and a constant $C>0$ independent of $y$ and $y_{0}$ such that $\left|y(x)-y_{0}(x)\right| \leq$ $C \sigma(x), x \in[a, b]$, then we say that the higher order integro-differential equation (1) has the $\sigma$-semi-Hyers-Ulam stability.

Some of the usual techniques to study the stability of functional, integral and integro-differential equations are a combination of fixed point results with a generalized metric in appropriate settings (cf. $[5,8,9,10,11,12,13,15])$. In view of this, and just for the sake of completeness, let us recall the definition of a generalized metric and the corresponding Banach Fixed Point Theorem.

Definition 2 Let $X$ a nonempty set. A function $d: X \times X \rightarrow[0,+\infty]$ is called a generalized metric on $X$ if and only if $d$ satisfies: (i) $d(x, y)=0$ if and only if $x=y$; (ii) $d(x, y)=d(y, x)$ for all $x, y \in X ;($ iii $) d(x, z) \leq d(x, y)+d(y, z)$ for all $x, y, z \in X$.

Theorem $3 \quad$ Let $(X, d)$ be a generalized complete metric space and $T: X \rightarrow X$ a strictly contractive operator with a Lipschitz constant $L<1$. If there exists a nonnegative integer $k$ such that $d\left(T^{k+1} x, T^{k} x\right)<\infty$ for some $x \in X$, then the following three propositions hold true: (i) the sequence $\left(T^{n} x\right)_{n \in \mathbb{N}}$ converges to a fixed point $x^{*}$ of $T$; (ii) $x^{*}$ is the unique fixed point of $T$ in $X^{*}=\left\{y \in X: d\left(T^{k} x, y\right)<\infty\right\}$; (iii) if $y \in X^{*}$, then

$$
d\left(y, x^{*}\right) \leq \frac{1}{1-L} d(T y, y) .
$$

\section{HYERS-ULAM-RASSIAS STABILITY IN THE FINITE INTERVAL CASE}

In this section we will present sufficient conditions for the Hyers-Ulam-Rassias stability of the higher order integrodifferential equation (1), where $x \in[a, b]$, for some fixed real numbers $a$ and $b$.

We will consider the space of continuously differentiable functions $C^{n}([a, b])$ on $[a, b]$ endowed with a generalization of the Bielecki metric

$$
d(u, v)=\sup _{x \in[a, b]} \frac{|u(x)-v(x)|}{\sigma(x)}
$$

where $\sigma$ is a non-decreasing continuous function $\sigma:[a, b] \rightarrow(0, \infty)$. If instead of $\sigma$ in (3) we have $e^{p(\cdot-a)}$ with $p>0$ then we have the well-known Bielecki metric.

We recall that $\left(C^{n}([a, b]), d\right)$ is a complete metric space (cf., [7], [27]).

Theorem 4 Let $\alpha:[a, b] \rightarrow[a, b]$ be a continuous delay function with $\alpha(t) \leq t$ for all $t \in[a, b]$ and $\sigma:[a, b] \rightarrow$ $(0, \infty)$ a non-decreasing continuous function. In addition, suppose that there is $\beta \in \mathbb{R}$ such that

$$
\int_{a}^{x} \sigma(\tau) d \tau \leq \beta \sigma(x)
$$


for all $x \in[a, b]$. Moreover, suppose that $f:[a, b] \times \mathbb{C} \times \mathbb{C} \rightarrow \mathbb{C}$ is a continuous function satisfying the Lipschitz condition $|f(x, u(x), g(x))-f(x, v(x), h(x))| \leq M(|u(x)-v(x)|+|g(x)-h(x)|)$ with $M>0$ and the kernel $k:[a, b] \times$ $[a, b] \times \mathbb{C} \times \mathbb{C} \rightarrow \mathbb{C}$ is a continuous function satisfying the Lipschitz condition $|k(x, t, u(t), u(\alpha(t)))-k(x, t, v(t), v(\alpha(t)))| \leq$ $L|u(\alpha(t))-v(\alpha(t))|$ with $L>0$.

If $y \in C^{n}([a, b])$ is such that

$$
\left|y^{(n)}(x)-f\left(x, y(x), \int_{a}^{x} k(x, \tau, y(\tau), y(\alpha(\tau))) d \tau\right)\right| \leq \sigma(x), \quad x \in[a, b],
$$

and $M\left(\beta^{n}+L \beta^{n+1}\right)<1$, then there is a unique function $y_{0} \in C^{n}([a, b])$ such that $y_{0}^{(n)}(x)=$ $f\left(x, y_{0}(x), \int_{a}^{x} k\left(x, \tau, y_{0}(\tau), y_{0}(\alpha(\tau))\right) d \tau\right)$ and

$$
\left|y(x)-y_{0}(x)\right| \leq \frac{\beta^{n}}{1-M\left(\beta^{n}+L \beta^{n+1}\right)} \sigma(x)
$$

for all $x \in[a, b]$. This means that under the above conditions, the higher order integro-differential equation (1) has the Hyers-Ulam-Rassias stability.

Proof. By integration we have that $y^{(n)}(x)=f\left(x, y(x), \int_{a}^{x} k(x, \tau, y(\tau), y(\alpha(\tau))) d \tau\right)$ is equivalent to $y(x)=$ $\int_{a}^{x} \int_{a}^{s_{n}} \int_{a}^{s_{n-1}} \ldots \int_{a}^{s_{2}} f\left(s_{1}, y\left(s_{1}\right), \int_{a}^{s_{1}} k\left(s_{1}, \tau, y(\tau), y(\alpha(\tau))\right) d \tau\right) d s_{1} \ldots d s_{n-2} d s_{n-1} d s_{n}$ where we are using the conditions $y^{(j)}(a)=0, j=0, \ldots, n-1$. So, we will consider the operator $T: C^{n}([a, b]) \rightarrow C^{n}([a, b])$, defined by

$$
(T u)(x)=\int_{a}^{x} \int_{a}^{s_{n}} \int_{a}^{s_{n-1}} \ldots \int_{a}^{s_{2}} f\left(s_{1}, u\left(s_{1}\right), \int_{a}^{s_{1}} k\left(s_{1}, \tau, u(\tau), u(\alpha(\tau))\right) d \tau\right) d s_{1} \ldots d s_{n-2} d s_{n-1} d s_{n},
$$

for all $x \in[a, b]$ and $u \in C^{n}([a, b])$.

Note that for any continuous function $u, T u$ is also continuous. Indeed, $\left|(T u)(x)-(T u)\left(x_{0}\right)\right|=$ $\left|\int_{x}^{x_{0}} \int_{a}^{s_{n}} \int_{a}^{s_{n-1}} \ldots \int_{a}^{s_{2}} f\left(s_{1}, u\left(s_{1}\right), \int_{a}^{s_{1}} k\left(s_{1}, \tau, u(\tau), u(\alpha(\tau))\right) d \tau\right) d s_{1} \ldots d s_{n-2} d s_{n-1} d s_{n}\right| \longrightarrow 0$, when $x \rightarrow x_{0}$. Note that by the hypothesis (4) we have

$$
\begin{gathered}
\int_{a}^{x} \int_{a}^{s_{n}} \int_{a}^{s_{n-1}} \ldots \int_{a}^{s_{2}} \int_{a}^{s_{1}} \sigma(\tau) d \tau d s_{1} \ldots d s_{n-2} d s_{n-1} d s_{n} \leq \int_{a}^{x} \int_{a}^{s_{n}} \int_{a}^{s_{n-1}} \ldots \int_{a}^{s_{2}} \beta \sigma\left(s_{1}\right) d s_{1} \ldots d s_{n-2} d s_{n-1} d s_{n} \\
\quad \leq \int_{a}^{x} \int_{a}^{s_{n}} \int_{a}^{s_{n-1}} \ldots \int_{a}^{s_{3}} \beta^{2} \sigma\left(s_{2}\right) d s_{2} \ldots d s_{n-2} d s_{n-1} d s_{n} \leq \ldots \leq \int_{a}^{x} \beta^{n} \sigma\left(s_{n}\right) d s_{n} \leq \beta^{n+1} \sigma(x) .
\end{gathered}
$$

Under the present conditions, we will deduce that the operator $T$ is strictly contractive with respect to the metric (3). Indeed, for all $u, v \in C^{n}([a, b])$, we have,

$$
\begin{aligned}
d(T u, T v)= & \sup _{x \in[a, b]} \frac{|(T u)(x)-(T v)(x)|}{\sigma(x)} \\
\leq & \sup _{x \in[a, b]} \frac{1}{\sigma(x)} \int_{a}^{x} \int_{a}^{s_{n}} \int_{a}^{s_{n-1}} \ldots \int_{a}^{s_{2}} \mid f\left(s_{1}, u\left(s_{1}\right), \int_{a}^{s_{1}} k\left(s_{1}, \tau, u(\tau), u(\alpha(\tau))\right) d \tau\right) \\
& \quad-f\left(s_{1}, v\left(s_{1}\right), \int_{a}^{s_{1}} k\left(s_{1}, \tau, v(\tau), v(\alpha(\tau))\right) d \tau\right) \mid d s_{1} \ldots d s_{n-2} d s_{n-1} d s_{n} \\
\leq & M \sup _{x \in[a, b]} \frac{1}{\sigma(x)} \int_{a}^{x} \int_{a}^{s_{n}} \int_{a}^{s_{n-1}} \ldots \int_{a}^{s_{2}}\left|u\left(s_{1}\right)-v\left(s_{1}\right)\right| d s_{1} \ldots d s_{n-2} d s_{n-1} d s_{n} \\
& \quad+M \sup _{x \in[a, b]} \frac{1}{\sigma(x)} \int_{a}^{x} \int_{a}^{s_{n}} \int_{a}^{s_{n-1}} \ldots \int_{a}^{s_{2}} \int_{a}^{s_{1}} \mid k\left(s_{1}, \tau, u(\tau), u(\alpha(\tau))\right) \\
& \quad-k\left(s_{1}, \tau, v(\tau), v(\alpha(\tau))\right) \mid d \tau d s_{1} \ldots d s_{n-2} d s_{n-1} d s_{n} \\
\leq & M \sup _{x \in[a, b]} \frac{1}{\sigma(x)} \int_{a}^{x} \int_{a}^{s_{n}} \int_{a}^{s_{n-1}} \ldots \int_{a}^{s_{2}}\left|u\left(s_{1}\right)-v\left(s_{1}\right)\right| d s_{1} \ldots d s_{n-2} d s_{n-1} d s_{n}
\end{aligned}
$$




$$
\begin{aligned}
& \quad+M L \sup _{x \in[a, b]} \frac{1}{\sigma(x)} \int_{a}^{x} \int_{a}^{s_{n}} \int_{a}^{s_{n-1}} \ldots \int_{a}^{s_{2}} \int_{a}^{s_{1}}|u(\alpha(\tau))-v(\alpha(\tau))| d \tau d s_{1} \ldots d s_{n-2} d s_{n-1} d s_{n} \\
& =M \sup _{x \in[a, b]} \frac{1}{\sigma(x)} \int_{a}^{x} \int_{a}^{s_{n}} \int_{a}^{s_{n-1}} \ldots \int_{a}^{s_{2}} \sigma\left(s_{1}\right) \frac{\left|u\left(s_{1}\right)-v\left(s_{1}\right)\right|}{\sigma\left(s_{1}\right)} d s_{1} \ldots d s_{n-2} d s_{n-1} d s_{n} \\
& \quad+M L \sup _{x \in[a, b]} \frac{1}{\sigma(x)} \int_{a}^{x} \int_{a}^{s_{n}} \int_{a}^{s_{n-1}} \ldots \int_{a}^{s_{2}} \int_{a}^{s_{1}} \sigma(\tau) \frac{|u(\alpha(\tau))-v(\alpha(\tau))|}{\sigma(\tau)} d \tau d s_{1} \ldots d s_{n-2} d s_{n-1} d s_{n} \\
& \leq M \sup _{s_{1} \in[a, b]} \frac{\left|u\left(s_{1}\right)-v\left(s_{1}\right)\right|}{\sigma\left(s_{1}\right)} \sup _{x \in[a, b]} \frac{1}{\sigma(x)} \int_{a}^{x} \int_{a}^{s_{n}} \int_{a}^{s_{n-1}} \ldots \int_{a}^{s_{2}} \sigma\left(s_{1}\right) d s_{1} \ldots d s_{n-2} d s_{n-1} d s_{n} \\
& \quad+M L \sup _{\tau \in[a, b]} \frac{|u(\tau)-v(\tau)|}{\sigma(\tau)} \sup _{x \in[a, b]} \frac{1}{\sigma(x)} \int_{a}^{x} \int_{a}^{s_{n}} \int_{a}^{s_{n-1}} \ldots \int_{a}^{s_{2}} \int_{a}^{s_{1}} \sigma(\tau) d \tau d s_{1} \ldots d s_{n-2} d s_{n-1} d s_{n} \\
& \leq M d(u, v) \beta^{n}+M L d(u, v) \sup _{x \in[a, b]} \frac{\beta^{n+1} \sigma(x)}{\sigma(x)} \\
& =M\left(\beta^{n}+L \beta^{n+1}\right) d(u, v) .
\end{aligned}
$$

Due to the fact that $M\left(\beta^{n}+L \beta^{n+1}\right)<1$ it follows that $T$ is strictly contractive. Thus, we can apply the above mentioned Banach Fixed Point Theorem, which ensures that we have the Hyers-Ulam-Rassias stability for the higher order integro-differential equation.

Additionally, (6) follows from (2) and (5). Indeed, from (5), we have

$$
-\sigma(x) \leq y^{(n)}(x)-f\left(x, y(x), \int_{a}^{x} k(x, \tau, y(\tau), y(\alpha(\tau))) d \tau\right) \leq \sigma(x), \quad x \in[a, b],
$$

and so, using integration, we obtain

$$
\begin{aligned}
& \left|y(x)-\int_{a}^{x} \int_{a}^{s_{n}} \int_{a}^{s_{n-1}} \ldots \int_{a}^{s_{2}} f\left(s_{1}, y\left(s_{1}\right), \int_{a}^{s_{1}} k\left(s_{1}, \tau, y(\tau), y(\alpha(\tau))\right) d \tau\right) d s_{1} \ldots d s_{n-2} d s_{n-1} d s_{n}\right| \\
& \leq \int_{a}^{x} \int_{a}^{s_{n}} \int_{a}^{s_{n-1}} \ldots \int_{a}^{s_{2}} \sigma\left(s_{1}\right) d s_{1} \ldots d s_{n-2} d s_{n-1} d s_{n} \leq \beta^{n} \sigma(x) .
\end{aligned}
$$

Therefore, having in mind (7) and (9), we conclude that

$$
|y(x)-(T y)(x)| \leq \beta^{n} \sigma(x), \quad x \in[a, b] .
$$

Now we can apply again the Banach Fixed Point Theorem and from (2) we have that

$$
d\left(y, y_{0}\right) \leq \frac{1}{1-M\left(\beta^{n}+L \beta^{n+1}\right)} d(T y, y) .
$$

From the definition of the metric $d$ and by (10) follows that

$$
\sup _{x \in[a, b]} \frac{\left|y(x)-y_{0}(x)\right|}{\sigma(x)} \leq \frac{\beta^{n}}{1-M\left(\beta^{n}+L \beta^{n+1}\right)}
$$

and consequently (6) holds.

\section{$\sigma$-SEMI-HYERS-ULAM AND HYERS-ULAM STABILITIES IN THE FINITE INTERVAL CASE}

In this section we will present sufficient conditions for the $\sigma$-semi-Hyers-Ulam stability and for the Hyers-Ulam stability of the higher order integro-differential equation (1). 
Theorem $5 \quad$ Let $\alpha:[a, b] \rightarrow[a, b]$ be a continuous delay function with $\alpha(t) \leq t$ for all $t \in[a, b]$ and $\sigma:[a, b] \rightarrow$ $(0, \infty)$ a non-decreasing continuous function. In addition, suppose that there is $\beta \in \mathbb{R}$ such that

$$
\int_{a}^{x} \sigma(\tau) d \tau \leq \beta \sigma(x)
$$

for all $x \in[a, b]$. Moreover, suppose that $f:[a, b] \times \mathbb{C} \times \mathbb{C} \rightarrow \mathbb{C}$ is a continuous function satisfying the Lipschitz condition $|f(x, u(x), g(x))-f(x, v(x), h(x))| \leq M(|u(x)-v(x)|+|g(x)-h(x)|)$ with $M>0$ and the kernel $k:[a, b] \times$ $[a, b] \times \mathbb{C} \times \mathbb{C} \rightarrow \mathbb{C}$ is a continuous function satisfying the Lipschitz condition $|k(x, t, u(t), u(\alpha(t)))-k(x, t, v(t), v(\alpha(t)))| \leq$ $L|u(\alpha(t))-v(\alpha(t))|$ with $L>0$.

If $y \in C^{n}([a, b])$ is such that

$$
\left|y^{(n)}(x)-f\left(x, y(x), \int_{a}^{x} k(x, \tau, y(\tau), y(\alpha(\tau))) d \tau\right)\right| \leq \theta, \quad x \in[a, b],
$$

where $\theta \geq 0$ and $M\left(\beta^{n}+L \beta^{n+1}\right)<1$, then there is a unique function $y_{0} \in C^{n}([a, b])$ such that $y_{0}^{(n)}(x)=$ $f\left(x, y_{0}(x), \int_{a}^{x} k\left(x, \tau, y_{0}(\tau), y_{0}(\alpha(\tau))\right) d \tau\right)$ and

$$
\left|y(x)-y_{0}(x)\right| \leq \frac{(b-a)^{n} \theta}{\left(1-M\left(\beta^{n}+L \beta^{n+1}\right)\right) \sigma(a)} \sigma(x)
$$

for all $x \in[a, b]$. This means that under the above conditions, the higher order integro-differential equation (1) has the $\sigma$-semi-Hyers-Ulam stability.

Proof. We will consider the operator $T: C^{n}([a, b]) \rightarrow C^{n}([a, b])$, defined by

$$
(T u)(x)=\int_{a}^{x} \int_{a}^{s_{n}} \int_{a}^{s_{n-1}} \ldots \int_{a}^{s_{2}} f\left(s_{1}, u\left(s_{1}\right), \int_{a}^{s_{1}} k\left(s_{1}, \tau, u(\tau), u(\alpha(\tau))\right) d \tau\right) d s_{1} \ldots d s_{n-2} d s_{n-1} d s_{n},
$$

for all $x \in[a, b]$ and $u \in C^{n}([a, b])$.

By the same procedure as above, we have that $T$ is strictly contractive with respect to the metric (3) due to the fact that $M\left(\beta^{n}+L \beta^{n+1}\right)<1$. Thus, we can apply the Banach Fixed Point Theorem, which ensures that we have the $\sigma$-semi-Hyers-Ulam stability for the higher order integro-differential equation (1).

On the other hand, from (14) and using integration we obtain

$$
\begin{aligned}
& \left|y(x)-\int_{a}^{x} \int_{a}^{s_{n}} \int_{a}^{s_{n-1}} \ldots \int_{a}^{s_{2}} f\left(s_{1}, y\left(s_{1}\right), \int_{a}^{s_{1}} k\left(s_{1}, \tau, y(\tau), y(\alpha(\tau))\right) d \tau\right) d s_{1} \ldots d s_{n-2} d s_{n-1} d s_{n}\right| \\
& \leq \int_{a}^{x} \int_{a}^{s_{n}} \int_{a}^{s_{n-1}} \ldots \int_{a}^{s_{2}} \theta d s_{1} \ldots d s_{n-2} d s_{n-1} d s_{n} \leq \theta(b-a)^{n} .
\end{aligned}
$$

Therefore, having in mind (16) and (17), we conclude that

$$
|y(x)-(T y)(x)| \leq \theta(b-a)^{n}, \quad x \in[a, b] .
$$

From (2), the definition of the metric $d$ and by (18) follows that

$$
\sup _{x \in[a, b]} \frac{\left|y(x)-y_{0}(x)\right|}{\sigma(x)} \leq \frac{1}{1-M\left(\beta^{n}+L \beta^{n+1}\right)} \sup _{x \in[a, b]} \frac{\theta(b-a)^{n}}{\sigma(x)} .
$$

and consequently by the definition of $\sigma$ we have that (15) holds.

Corollary $6 \quad$ Let $\alpha:[a, b] \rightarrow[a, b]$ be a continuous delay function with $\alpha(t) \leq t$ for all $t \in[a, b]$ and $\sigma:[a, b] \rightarrow$ $(0, \infty)$ a non-decreasing continuous function. In addition, suppose that there is $\beta \in \mathbb{R}$ such that

$$
\int_{a}^{x} \sigma(\tau) d \tau \leq \beta \sigma(x)
$$


for all $x \in[a, b]$. Moreover, suppose that $f:[a, b] \times \mathbb{C} \times \mathbb{C} \rightarrow \mathbb{C}$ is a continuous function satisfying the Lipschitz condition $|f(x, u(x), g(x))-f(x, v(x), h(x))| \leq M(|u(x)-v(x)|+|g(x)-h(x)|)$ with $M>0$ and the kernel $k:[a, b] \times$ $[a, b] \times \mathbb{C} \times \mathbb{C} \rightarrow \mathbb{C}$ is a continuous function satisfying the Lipschitz condition

$$
|k(x, t, u(t), u(\alpha(t)))-k(x, t, v(t), v(\alpha(t)))| \leq L|u(\alpha(t))-v(\alpha(t))|
$$

with $L>0$.

If $y \in C^{n}([a, b])$ is such that

$$
\left|y^{(n)}(x)-f\left(x, y(x), \int_{a}^{x} k(x, \tau, y(\tau), y(\alpha(\tau))) d \tau\right)\right| \leq \theta, \quad x \in[a, b],
$$

where $\theta \geq 0$ and $M\left(\beta^{n}+L \beta^{n+1}\right)<1$, then there is a unique function $y_{0} \in C^{n}([a, b])$ such that $y_{0}^{(n)}(x)=$ $f\left(x, y_{0}(x), \int_{a}^{x} k\left(x, \tau, y_{0}(\tau), y_{0}(\alpha(\tau))\right) d \tau\right)$ and

$$
\left|y(x)-y_{0}(x)\right| \leq \frac{(b-a)^{n} \sigma(b)}{\left(1-M\left(\beta^{n}+L \beta^{n+1}\right)\right) \sigma(a)} \theta
$$

for all $x \in[a, b]$. This means that under the above conditions, the higher order integro-differential equation (1) has the Hyers-Ulam stability.

\section{HYERS-ULAM-RASSIAS STABILITY IN THE INFINITE INTERVAL CASE}

In this section, we analyse the Hyers-Ulam-Rassias stability of the higher order integro-differential equation (1) but, instead of considering a finite interval $[a, b]$ (with $a, b \in \mathbb{R}$ ), we will consider the infinite interval $[a, \infty$ ), for some fixed $a \in \mathbb{R}$.

Thus, we will now be dealing with the higher order integro-differential equations,

$$
y^{(n)}(x)=f\left(x, y(x), \int_{a}^{x} k(x, \tau, y(\tau), y(\alpha(\tau))) d \tau\right), \quad y^{(j)}(a)=0, j=0, \ldots, n-1,
$$

with $y \in C^{n}([a, \infty)), x \in[a, \infty)$ where $a$ is a fixed real number, $f:[a, \infty) \times \mathbb{C} \times \mathbb{C} \rightarrow \mathbb{C}$ and $k:[a, \infty) \times[a, \infty) \times \mathbb{C} \times \mathbb{C} \rightarrow \mathbb{C}$ are continuous functions, and $\alpha:[a, \infty) \rightarrow[a, \infty)$ is a continuous delay function which therefore fulfills $\alpha(\tau) \leq \tau$ for all $\tau \in[a, \infty)$.

Our strategy will be based on a recurrence procedure due to the already obtained result for the corresponding finite interval case.

Let us consider a fixed non-decreasing continuous function $\sigma:[a, \infty) \rightarrow(\varepsilon, \omega)$, for some $\varepsilon, \omega>0$ and the space $C_{b}^{n}([a, \infty))$ of bounded differentiable functions endowed with the metric

$$
d_{b}(u, v)=\sup _{x \in[a, \infty)} \frac{|u(x)-v(x)|}{\sigma(x)}
$$

Theorem $7 \quad$ Let $\alpha:[a, \infty) \rightarrow[a, \infty)$ be a continuous delay function with $\alpha(t) \leq t$ for all $t \in[a, \infty)$ and $\sigma$ : $[a, \infty) \rightarrow(\varepsilon, \omega)$, for some $\varepsilon, \omega>0$, a non-decreasing continuous function. In addition, suppose that there is $\beta \in \mathbb{R}$ such that $\int_{a}^{x} \sigma(\tau) d \tau \leq \beta \sigma(x)$, for all $x \in[a, \infty)$. Moreover, suppose that $f:[a, \infty) \times \mathbb{C} \times \mathbb{C} \rightarrow \mathbb{C}$ is a continuous function satisfying the Lipschitz condition $|f(x, u(x), g(x))-f(x, v(x), h(x))| \leq M(|u(x)-v(x)|+|g(x)-h(x)|)$ with $M>0$ and the kernel $k:[a, \infty) \times[a, \infty) \times \mathbb{C} \times \mathbb{C} \rightarrow \mathbb{C}$ is a continuous function so that $\int_{a}^{x} k(x, \tau, z(\tau), z(\alpha(\tau))) d \tau$ is a bounded continuous function for any bounded continuous function $z$. In addition, suppose that $k$ satisfies the Lipschitz condition $|k(x, t, u(t), u(\alpha(t)))-k(x, t, v(t), v(\alpha(t)))| \leq L|u(\alpha(t))-v(\alpha(t))|$ with $L>0$.

If $y \in C_{b}^{n}([a, \infty))$ is such that

$$
\left|y^{(n)}(x)-f\left(x, y(x), \int_{a}^{x} k(x, \tau, y(\tau), y(\alpha(\tau))) d \tau\right)\right| \leq \sigma(x), \quad x \in[a, \infty),
$$


and $M\left(\beta^{n}+L \beta^{n+1}\right)<1$, then there is a unique function $y_{0} \in C_{b}^{n}([a, \infty))$ such that

$$
y_{0}^{(n)}(x)=f\left(x, y_{0}(x), \int_{a}^{x} k\left(x, \tau, y_{0}(\tau), y_{0}(\alpha(\tau))\right) d \tau\right)
$$

and

$$
\left|y(x)-y_{0}(x)\right| \leq \frac{\beta^{n}}{1-M\left(\beta^{n}+L \beta^{n+1}\right)} \sigma(x)
$$

for all $x \in[a, \infty)$. This means that under the above conditions, the higher order integro-differential equation (24) has the Hyers-Ulam-Rassias stability.

Proof. For any $m \in \mathbb{N}$, we will define $I_{m}=[a, a+m]$. By Theorem 4 , there exists a unique bounded differentiable function $y_{0, m}: I_{m} \rightarrow \mathbb{C}$ such that

$$
y_{0, m}^{(n)}(x)=f\left(x, y_{0, m}(x), \int_{a}^{x} k\left(x, \tau, y_{0, m}(\tau), y_{0, m}(\alpha(\tau))\right) d \tau\right)
$$

and

$$
\left|y(x)-y_{0, m}(x)\right| \leq \frac{\beta^{n}}{1-M\left(\beta^{n}+L \beta^{n+1}\right)} \sigma(x)
$$

for all $x \in I_{m}$. The uniqueness of $y_{0, m}$ implies that if $x \in I_{m}$ then

$$
y_{0, m}(x)=y_{0, m+1}(x)=y_{0, m+2}(x)=\cdots .
$$

For any $x \in[a, \infty)$, let us define $m(x) \in \mathbb{N}$ as $m(x)=\min \left\{m \in \mathbb{N}: x \in I_{m}\right\}$. We also define a function $y_{0}:[a, \infty) \rightarrow \mathbb{C}$ by

$$
y_{0}(x)=y_{0, m(x)}(x) .
$$

For any $x_{1} \in[a, \infty)$, let $m_{1}=m\left(x_{1}\right)$. Then $x_{1} \in$ Int $I_{m_{1}+1}$ and there exists an $\epsilon>0$ such that $y_{0}(x)=y_{0, m_{1}+1}(x)$ for all $x \in\left(x_{1}-\epsilon, x_{1}+\epsilon\right)$. By Theorem 4, $y_{0, m_{1}+1}$ is continuous at $x_{1}$, and so it is $y_{0}$.

Now, we will prove that $y_{0}$ satisfies

$$
y_{0}^{(n)}(x)=f\left(x, y_{0}(x), \int_{a}^{x} k\left(x, \tau, y_{0}(\tau), y_{0}(\alpha(\tau))\right) d \tau\right)
$$

and

$$
\left|y(x)-y_{0}(x)\right| \leq \frac{\beta^{n}}{1-M\left(\beta^{n}+L \beta^{n+1}\right)} \sigma(x) .
$$

For an arbitrary $x \in[a, \infty)$ we choose $m(x)$ such that $x \in I_{m(x)}$. By (29) and (32), we have

$$
\begin{aligned}
y_{0}(x)=y_{0, m(x)}(x) & =f\left(x, y_{0, m(x)}(x), \int_{a}^{x} k\left(x, \tau, y_{0, m(x)}(\tau), y_{0, m(x)}(\alpha(\tau))\right) d \tau\right) \\
& =f\left(x, y_{0}(x), \int_{a}^{x} k\left(x, \tau, y_{0}(\tau), y_{0}(\alpha(\tau))\right) d \tau\right) .
\end{aligned}
$$

Note that $m(\tau) \leq m(x)$, for any $\tau \in I_{m(x)}$, and it follows from $(31)$ that $y_{0}(\tau)=y_{0, m(\tau)}(\tau)=y_{0, m(x)}(\tau)$, so, the last equality in (35) holds.

To prove (28), by (32) and (30), we have that for all $x \in[a, \infty),\left|y(x)-y_{0}(x)\right|=\left|y(x)-y_{0, m(x)}(x)\right| \leq$ $\frac{\beta^{n}}{1-M\left(\beta^{n}-L \beta^{n+1}\right)} \sigma(x)$. Finally, we will prove the uniqueness of $y_{0}$. Let us consider another bounded differentiable function $y_{1}$ which satisfies (27) and (28), for all $x \in[a, \infty)$. By the uniqueness of the solution on $I_{m(x)}$ for any $m(x) \in \mathbb{N}$ we have that $y_{0 \mid I_{m(x)}}=y_{0, m(x)}$ and $y_{1 \mid I_{m(x)}}$ satisfies (27) and (28) for all $x \in I_{m(x)}$, so $y_{0}(x)=\left.y_{0}\right|_{I_{m(x)}}(x)=\left.y_{1}\right|_{I_{m(x)}}(x)=y_{1}(x)$.

Remark 8 With the necessary adaptations, Theorem 7 also holds true for infinite intervals of the type $(-\infty, b]$, with $b \in \mathbb{R}$, as well as for $(-\infty, \infty)$. 


\section{Illustrative Examples}

In this section we will present some examples simply to illustrate that the conditions of the above results are possible to attain.

For 2-differentiable functions $y:[0,1] \rightarrow \mathbb{R}$, let us start be considering the integro-differential equation

$$
y^{\prime \prime}(x)=-2 y(x)+2+\int_{0}^{x}((\tau-x) y(\alpha(\tau))) d \tau, \quad x \in[0,1], y^{(j)}(0)=0, j=0,1
$$

as well as the continuous function $\sigma:[0,1] \rightarrow(0, \infty)$ defined by $\sigma(x)=1.1 e^{10 x}$ and the continuous function $\alpha:[0,1] \rightarrow[0,1]$ given by $\alpha(x)=x$.

We have all the conditions of Theorem 5 being satisfied. In fact, such $\alpha:[0,1] \rightarrow[0,1]$ defined by $\alpha(x)=x$ is a continuous function, and obviously $\alpha(x) \leq x$. Moreover, for $\beta=0.1\left(1-e^{-10}\right)$ we have that $\sigma:[0,1] \rightarrow(0, \infty)$ defined by $\sigma(x)=1.1 e^{10 x}$ is a continuous function fulfilling

$$
\int_{0}^{x} 1.1 e^{10 \tau} d \tau \leq \beta 1.1 e^{10 x}=0.1\left(1-e^{-10}\right) \sigma(x), \quad x \in[0,1]
$$

$f:[0,1] \times \mathbb{C} \times \mathbb{C} \rightarrow \mathbb{C}$ defined by $f(x, y(x), g(x))=-2 y(x)+2+g(x)$ is a continuous function which fulfills $|f(x, u(x), g(x))-f(x, v(x), h(x))| \leq 2(|u(x)-v(x)|+|g(x)-h(x)|), x \in[0,1]$ (and so the previous constant $M$ is here taking the value 2); the kernel $k:[0,1] \times[0,1] \times \mathbb{C} \times \mathbb{C} \rightarrow \mathbb{C}$ defined by $k(x, \tau, y(\tau), y(\alpha(\tau)))=(\tau-x) y(\alpha(\tau))$ is a continuous function which fulfils the condition $|k(x, \tau, u(\tau), u(\alpha(\tau)))-k(x, \tau, v(\tau), v(\alpha(\tau)))| \leq|u(\alpha(\tau))-v(\alpha(\tau))|$, $\tau \in[0, x], x \in[0,1]$ (where we may identify 1 as the constant previously denoted by $L$ ). Thus, $M\left(\beta^{2}+L \beta^{3}\right) \simeq$ $0.021998<1$.

If we choose $y(x)=100 / 99 x \sin x$, it follows $\left|y^{\prime \prime}(x)-f\left(x, y(x), \int_{0}^{x} k(x, \tau, y(\tau), y(\alpha(\tau))) d \tau\right)\right|=\frac{2}{99}=: \theta, x \in[0,1]$.

Therefore, from Theorem 5, we have the $\sigma$-semi-Hyers-Ulam stability of the integro-differential equation (36), and from Corollary 6 we have the consequent Hyers-Ulam stability. In particular, having in mind the exact solution $y_{0}(x)=x \sin x$ of (36), it follows that $\left|y(x)-y_{0}(x)\right|=\left|\frac{1}{99} x \sin x\right| \leq \frac{\theta}{\left(1-2\left(\beta^{2}+\beta^{3}\right)\right) \sigma(0)} \sigma(x), x \in[0,1]$, and $\left|y(x)-y_{0}(x)\right|=$ $\left|\frac{1}{99} x \sin x\right| \leq \frac{\sigma(1)}{\left(1-2\left(\beta^{2}+\beta^{3}\right)\right) \sigma(0)} \theta, x \in[0,1]$.

Still within this first example associated with the integro-differential equation (36), and using the same $M$ and $L$, we will now show that all the conditions of Theorem 4 being satisfied. Indeed, let us now consider for $\sigma$ the continuous function $\sigma:[0,1] \rightarrow[0, \infty)$ defined by $\sigma(x)=0.0002+0.007 x+0.03 x^{2}$. If this is the case, instead of (37), we have now

$$
\int_{0}^{x} 0.0002+0.007 \tau+0.03 \tau^{2} d \tau \leq 0.36828\left(0.0002+0.007 x+0.03 x^{2}\right)=\beta \sigma(x), \quad x \in[0,1],
$$

and so still having $M\left(\beta^{2}+L \beta^{3}\right) \simeq 0.37116<1$.

Considering now $y(x)=0.99 x \sin (1.01 x)$, it follows

$$
\begin{aligned}
\left|y^{\prime \prime}(x)-f\left(x, y(x), \int_{0}^{x} k(x, \tau, y(\tau), y(\alpha(\tau))) d \tau\right)\right| & =\left|\frac{401979699}{5151505000} \cos (1.01 x)-\frac{3999699}{10201000000} x \sin (1.01 x)-\frac{80602}{1030301}\right| \\
& \leq \sigma(x),
\end{aligned}
$$

for all $x \in[0,1]$, and so we have the Hyers-Ulam-Rassias stability of the integro-differential equation (36). Having in mind the exact solution $y_{0}(x)=x \sin x$ of (36), it follows that

$$
\left|y(x)-y_{0}(x)\right|=|0.99 x \sin (1.01 x)-x \sin x| \leq \frac{\beta^{2}}{1-2\left(\beta^{2}+\beta^{3}\right)} \sigma(x), \quad x \in[0,1] .
$$

Let us now turn to other example in which the Hyers-Ulam-Rassias stability is illustrated. For a 2-differentiable function $y:[0.1,1] \rightarrow \mathbb{R}$, we shall consider the integro-differential equation

$$
y^{\prime \prime}(x)=2-\frac{x}{12} y(x) \sin x+\frac{1}{12000} \sin x+\int_{0.1}^{x}(\sin x y(\alpha(\tau))) d \tau, \quad x \in[0.1,1], y^{(j)}(0)=0, j=0,1
$$



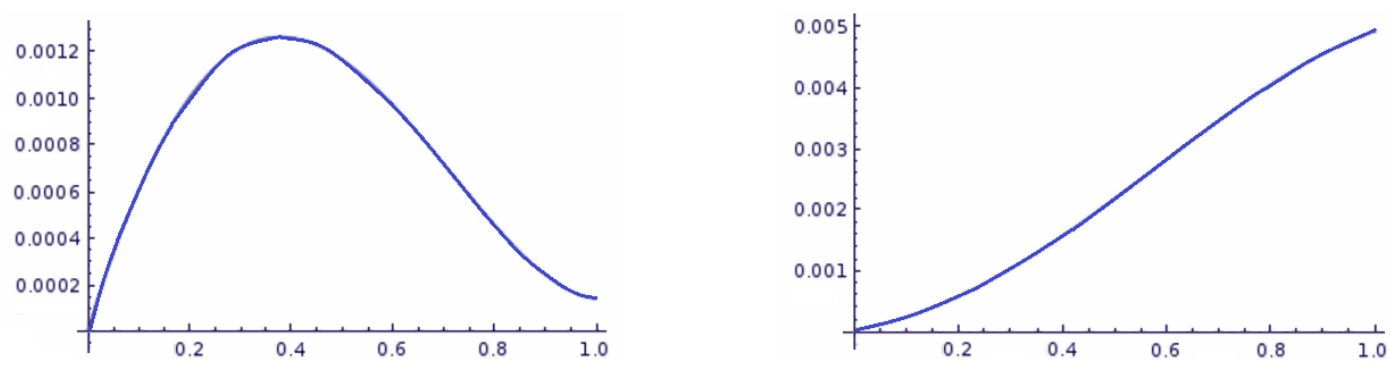

FIGURE 1. On the left, we have $z_{1}(x)=\sigma(x)-\left|y^{\prime \prime}(x)-f\left(x, y(x), \int_{0}^{x} k(x, \tau, y(\tau), y(\alpha(\tau))) d \tau\right)\right|$ to illustrate the inequality (39); on the right, we have $z_{2}(x)=\frac{\beta^{2}}{1-2\left(\beta^{2}+\beta^{3}\right)} \sigma(x)-\left|y(x)-y_{0}(x)\right|$ to illustrate the inequality (40).

as well as the continuous function $\sigma:[0.1,1] \rightarrow(0, \infty)$ defined by $\sigma(x)=0.02 e^{0.9 x}$ and the continuous delay function $\alpha:[0.1,1] \rightarrow[0.05,0.5]$ given by $\alpha(x)=x / 2$.

We realize that all the conditions of Theorem 4 are here satisfied. In fact, $\alpha(x)=x / 2$ is obviously continuous and satisfies $\alpha(x) \leq x$; for e.g. $\beta=0.617, \sigma:[0.1,1] \rightarrow[0, \infty)$ defined by $\sigma(x)=0.02 e^{0.9 x}$ is a continuous function which fulfills $\int_{0.1}^{x} 0.02 e^{0.9 \tau} d \tau \leq 0.617 \times 0.02 e^{0.9 x}=\beta \sigma(x), x \in[0.1,1] ; f:[0.1,1] \times \mathbb{C} \times \mathbb{C} \rightarrow \mathbb{C}$ defined by $f(x, y(x), g(x))=2-\frac{x}{12} y(x) \sin x+\frac{1}{12000} \sin x+g(x)$ is a continuous function which fulfills $\mid f(x, u(x), g(x))-$ $f(x, v(x), h(x))|\leq| u(x)-v(x)|+| g(x)-h(x) \mid, x \in[0.1,1]$ (where we may identify $M=1$ ); the kernel $k:[0.1,1] \times$ $[0.1,1] \times \mathbb{C} \times \mathbb{C} \rightarrow \mathbb{C}$ defined by $k(x, \tau, y(\tau), y(\alpha(\tau)))=\sin x y(\alpha(\tau))$ is a continuous function which fulfils the condition $|k(x, \tau, u(\tau), u(\alpha(\tau)))-k(x, \tau, v(\tau), v(\alpha(\tau)))| \leq \sin 1|u(\alpha(\tau))-v(\alpha(\tau))|, \tau \in[0.1, x], x \in[0.1,1]$, where we make the identification $L=\sin 1$. Thus, $M\left(\beta^{2}+L \beta^{3}\right) \simeq 0.578338<1$. Moreover, if we choose $y(x)=0.99 x^{2}$, it follows,

$$
\left|y^{\prime \prime}(x)-f\left(x, y(x), \int_{0}^{x} k(x, \tau, y(\tau), y(\alpha(\tau))) d \tau\right)\right|=\left|-0.02-8 .(3) \times 10^{-7} \sin x\right| \leq \sigma(x), \quad x \in[0.1,1] .
$$

Therefore, the integro-differential equation (41) exhibits the Hyers-Ulam-Rassias stability. In particular, by using the exact solution $y_{0}(x)=x^{2}$, we realize that

$$
\left|y(x)-y_{0}(x)\right|=0.01 x^{2} \leq \frac{\beta^{2}}{1-\left(\beta^{2}+\sin 1 \beta^{3}\right)} \sigma(x) .
$$
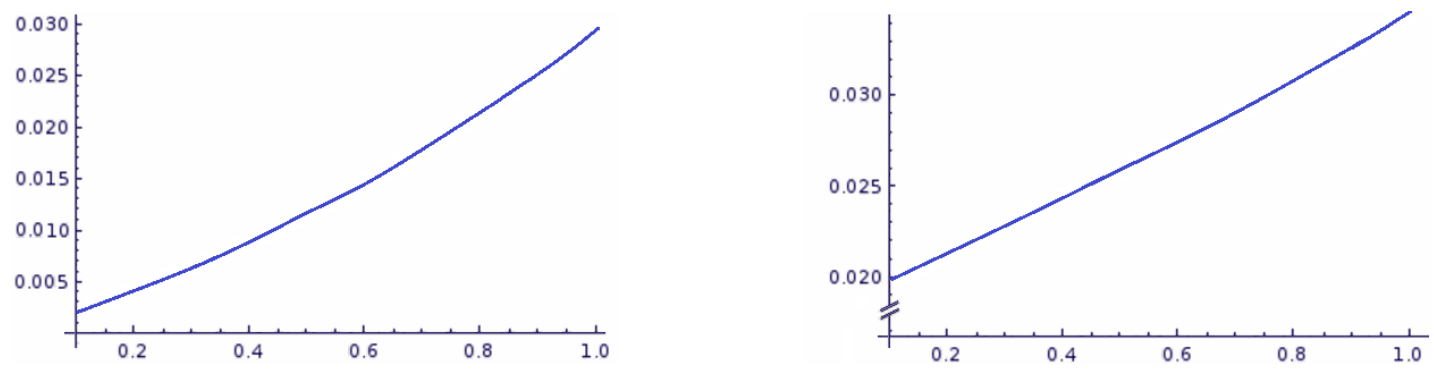

FIGURE 2. On the left, we have $z_{3}(x)=\sigma(x)-\left|y^{\prime \prime}(x)-f\left(x, y(x), \int_{0.1}^{x} k(x, \tau, y(\tau), y(\alpha(\tau))) d \tau\right)\right|$ to illustrate the inequality (42); on the right, we have $z_{4}(x)=\frac{\beta^{2}}{1-\left(\beta^{2}+\sin 1 \beta^{3}\right)} \sigma(x)-\left|y(x)-y_{0}(x)\right|$ to illustrate the inequality (43).

\section{ACKNOWLEDGMENTS}

This work was supported in part by FCT-Portuguese Foundation for Science and Technology through the Center for Research and Development in Mathematics and Applications (CIDMA) of University of Aveiro, within UID/MAT/04106/2013, and through the Center of Mathematics and Applications of University of Beira Interior (CMA-UBI), within project UID/MAT/00212/2013. 


\section{REFERENCES}

[1] H. H. Alsulami, S. Gülyaz, E. Karapinar and I. M. Erhan, "An Ulam stability result on quasi-b-metric-like spaces", Open Math. 14, 1087-1103 (2016).

[2] T. Aoki, "On the stability of the linear transformation in Banach spaces", J. Math. Soc. Japan 2, 64-66 (1950).

[3] A. Bahyrycz, J. Brzdȩk, E. Jablońska and R. Malejki, "Ulam's stability of a generalization of the Fréchet functional equation", J. Math. Anal. Appl. 442(2), 537-553 (2016).

[4] N. B.-Belluot, J. Brzdęk and K. Ciepliński, "On some recent developments in Ulam's type stability", Abstr. Appl. Anal. 2012, 41 (2012).

[5] J. Brzdęk, L. Cădariu and K. Ciepliński, "Fixed point theory and the Ulam stability", J. Funct. Spaces 2014, 16 (2014).

[6] T. A. Burton, Volterra Integral and Differential Equations (Elsevier, Amsterdam, 2005).

[7] L. Cădariu, L. Găvruţa and P. Găvruça, "Weighted space method for the stability of some nonlinear equations", Appl. Anal. Discrete Math. 6(1), 126-139 (2012).

[8] L. P. Castro and R. C. Guerra, "Hyers-Ulam-Rassias stability of Volterra integral equations within weighted spaces", Lib. Math. (N.S.) 33(2), 21-35 (2013).

[9] L. P. Castro and A. Ramos, "Hyers-Ulam and Hyers-Ulam-Rassias stability of Volterra integral equations with a delay", in Integral Methods in Science and Engineering, edited by C. Constanda et al., (Birkhäuser, Basel, 2010), 85-94.

[10] L. P. Castro and A. Ramos, "Hyers-Ulam-Rassias stability for a class of nonlinear Volterra integral equations", Banach J. Math. Anal. 3(1), 36-43 (2009).

[11] L. P. Castro and A. Ramos, "Hyers-Ulam stability for a class of Fredholm integral equations", in Mathematical Problems in Engineering Aerospace and Sciences ICNPAA 2010, Proceedings of the 8th International Conference of Mathematical Problems in Engineering, Aerospace and Science, edited by S. Sivasundaram (Cambridge Scientific Publishers, Cambridge, 2011), 171-176.

[12] L. P. Castro and A. M. Simões, "Hyers-Ulam and Hyers-Ulam-Rassias stability of a class of Hammerstein integral equations", AIP Conference Proceedings, 1798:020036-1/10 (2017).

[13] L. P. Castro and A. M. Simões, "Hyers-Ulam and Hyers-Ulam-Rassias stability of a class of integral equations on finite intervals", in CMMSE'17: Proceedings of the 17th International Conference on Computational and Mathematical Methods in Science and Engineering, edited by J. Vigo-Aguiar (@CMMSE, Costa Ballena, Rota, 2017), 507-515.

[14] L. P. Castro and A. M. Simões, "Different types of Hyers-Ulam-Rassias stabilities for a class of integrodifferential equations", Filomat 31(17), 5379-5390 (2017).

[15] L. P. Castro and A. M. Simões, "Hyers-Ulam and Hyers-Ulam-Rassias stability for a class of integrodifferential equations" in Mathematical Methods in Engineering: Theoretical Aspects, edited by K. Tas, D. Baleanu and J. A. Tenreiro Machado, Springer (in press).

[16] L. P. Castro and A. M. Simões, "Hyers-Ulam-Rassias stability of nonlinear integral equations through the Bielecki metric", Mathematical Methods in the Applied Sciences, 1-17 (2018).

[17] Y. J. Cho, C. Park, T. M. Rassias and R. Saadati, Stability of Functional Equations in Banach Algebras (Springer International Publishing, Switzerland, 2015).

[18] W.-S. Du, "A generalization of Diaz-Margolis's fixed point theorem and its application to the stability of generalized Volterra integral equations", J. Inequal. Appl. 2015:407, 15 (2015).

[19] Z. Gajda, "On stability of additive mappings", Int. J. Math. Math. Sci. 14(3), 431-434 (1991).

[20] A. M. Hassan, E. Karapinar and H. H. Alsulami, "Ulam-Hyers stability for MKC mappings via fixed point theory", Journal of Function Spaces 2016, 11 (2016).

[21] D. H. Hyers, "On the stability of linear functional equation", Proc. Natl. Acad. Sci. 27(4), 222-224 (1941).

[22] D. H. Hyers, G. Isac and Th. M. Rassias, Stability of Functional Equations in Several Variables (Birkhäuser, Basel, 1998).

[23] S.-M. Jung, Hyers-Ulam-Rassias Stability of Functional Equations in Mathematical Analysis (Hadronic Press, Palm Harbor, 2001).

[24] D. Popa and I. Raşa, "On the best constant in Hyers-Ulam stability of some positive linear operators", J. Math. Anal. Appl. 412(1), 103-108 (2014).

[25] Th. M. Rassias, "On the stability of the linear mapping in Banach spaces", Proc. Amer. Math. Soc. 72, 297-300 (1978).

[26] Th. M. Rassias, "On a modified Hyers-Ulam sequence”, J. Math. Anal. Appl. 158(1), 106-113 (1991).

[27] C. C. Tisdell and A. Zaidi, "Basic qualitative and quantitative results for solutions to nonlinear, dynamic equations on time scales with an application to economic modelling", Nonlinear Anal. 68(1), 3504-3524 (2008).

[28] S. M. Ulam, A Collection of the Mathematical Problems (Interscience Publication, New York, 1960). 